\title{
Video Article \\ Assessment of Social Cognition in Non-human Primates Using a Network of Computerized Automated Learning Device (ALDM) Test Systems
}

\author{
Joël Fagot ${ }^{1}$, Yousri Marzouki ${ }^{1}$, Pascal Huguet ${ }^{1}$, Julie Gullstrand ${ }^{1}$, Nicolas Claidière ${ }^{1}$ \\ ${ }^{1}$ CNRS, Federation $3 \mathrm{C}$, and Laboratory of Cognitive Psychology, Aix-Marseille University \\ Correspondence to: Joël Fagot at joel.fagot@univ-amu.fr
}

URL: https://www.jove.com/video/52798

DOI: doi: $10.3791 / 52798$

Keywords: Behavior, Issue 99, Baboon, automated learning device, cultural transmission, emotion, social facilitation, cognition, operant conditioning.

\section{Date Published: 5/5/2015}

Citation: Fagot, J., Marzouki, Y., Huguet, P., Gullstrand, J., Claidière, N. Assessment of Social Cognition in Non-human Primates Using a Network of Computerized Automated Learning Device (ALDM) Test Systems. J. Vis. Exp. (99), e52798, doi:10.3791/52798 (2015).

\section{Abstract}

Fagot \& Paleressompoulle ${ }^{1}$ and Fagot \& Bonte ${ }^{2}$ have published an automated learning device (ALDM) for the study of cognitive abilities of monkeys maintained in semi-free ranging conditions. Data accumulated during the last five years have consistently demonstrated the efficiency of this protocol to investigate individual/physical cognition in monkeys, and have further shown that this procedure reduces stress level during animal testing ${ }^{3}$. This paper demonstrates that networks of ALDM can also be used to investigate different facets of social cognition and ingroup expressed behaviors in monkeys, and describes three illustrative protocols developed for that purpose. The first study demonstrates how ethological assessments of social behavior and computerized assessments of cognitive performance could be integrated to investigate the effects of socially exhibited moods on the cognitive performance of individuals. The second study shows that batteries of ALDM running in parallel can provide unique information on the influence of the presence of others on task performance. Finally, the last study shows that networks of ALDM test units can also be used to study issues related to social transmission and cultural evolution. Combined together, these three studies demonstrate clearly that ALDM testing is a highly promising experimental tool for bridging the gap in the animal literature between research on individual cognition and research on social cognition.

\section{Video Link}

The video component of this article can be found at https://www.jove.com/video/52798/

\section{Introduction}

Experimental laboratory investigations of primate cognition have almost systematically favored the test of isolated animals ${ }^{4}$, depending on the issue tackled. This classical laboratory procedure has demonstrated its efficiency, and most of our knowledge on issues such as perception, memory, reasoning and abstract conceptualization comes from the test of isolated subjects. Testing isolated individuals conveys many practical advantages; this approach facilitates complex experimental designs combined with highly rigorous measurements (e.g., msec timing) of the behavior that would be otherwise inaccessible. However, this procedure also has its limits, when it comes to the study of social influences on cognition in particular. Researchers are often forced to leave the sophisticated laboratory environment in favor of large enclosures or wild contexts when their study involves social interactions among individuals. This choice has a cost as it is much more difficult to control the experimental factors and to measure their effects with the same level of precision outside of the laboratory. Understanding how real social interactions influence cognition is one of the most difficult scientific challenges in both humans and nonhuman primates.

The present paper presents a new experimental protocol aimed at alleviating this general difficulty to investigate social cognition in laboratory based nonhuman primates. Fagot \& Paleressompoulle ${ }^{1}$ and Fagot \& Bonte ${ }^{2}$ have recently published an automated learning device (ALDM) for the assessment of cognitive abilities of monkeys raised in semi-free conditions. This method combines the operant conditioning techniques traditionally used in laboratories with a Radio Frequency Identification (RFID) technology. In practice, monkeys living in their social group have a free access from their outdoor enclosure to several ALDM test units. ADLMs consist of open test systems containing all of the necessary equipment traditionally available in laboratories, in particular a touch screen to display the stimulus and record the response and a food dispenser to deliver the reward. A RFID microchip reader captures the numeric identity of the participant when it enters an ALDM test unit, and the test program uses this information to present the research program assigned to this particular subject.

Evidence accumulated during the past 5 years has clearly revealed that (1) ALDM testing enhances the motivation of the participants ${ }^{1,2}$ and (2) favors complex learning ${ }^{5,6}$. ALDM testing also seems beneficial in terms of animal welfare, and reduces the stress level of the participant ${ }^{3}$, see also $^{7}$. The ALDM testing procedure inspired the development of test devices by several research groups working with crested (Macaca nigra) and rhesus (Macaca mulatta) macaques ${ }^{7,8}$ and pigeons (Columba livia) ${ }^{9}$. The main goal of our paper is to demonstrate the potential contribution of ALDM testing for studies in the domain of social cognition. Three different uses of this protocol are described for that purpose. The ALDM test units have already been described in details in Fagot \& Paleressompoulle ${ }^{1}$. They are illustrated in Figure 1 and described briefly below. ALDM test units are open access operant conditioning test systems equipped with a Radio Frequency Identification (RFID) system that reads the RFID 
microchip of the monkey, and therefore its identity, once it enters the test system. The stimuli are presented on a touch screen, and food rewards are delivered inside the test system after a correct response is given (choice of the correct stimulus) to these stimuli.

\section{Protocol}

Ethics statement: This research was carried out in accordance with French standards and received approval from the national French ethics committee, the "Comite d'Ethique CE-14 pour l'Expérimentation Animale". Procedures were also consistent with the guidelines of the Association for the Study of Animal Behaviour.

\section{ALDM Test Unit Set-up}

NOTE: Set up the ALDM test units as per Fagot \& Paleressompoulle ${ }^{1}$.

1. Create test chambers of dimension $\left(0.7 \times 0.7 \times 0.8 \mathrm{~m}^{3}\right)$ as described in ${ }^{1}$. Ensure that each test chamber has 2 lateral transparent sidewalls. Equip each test chamber with a sliding door that can be open or closed on the backside (see Figure 1). Open the back door of the ALDM unit to provide access to the test system from the enclosure.

2. Fit the front panel of the test chamber with a $\left(7.3 \times 7 \mathrm{~cm}^{2}\right)$ view port providing vision of a 19 in touch screen located in front of the unit, and 2 handports for manual interactions with the touch screen.

3. Install an antenna connected to a microchip reader around each hand port as described in ${ }^{1}$. The antenna will capture the RFID identity of the subject.

4. Install a 19 in capacitive touch screen in front of each test chamber. The touchscreen serves to present the stimuli and to record response related variables (accuracy and response time). Separate the viewports $25 \mathrm{~cm}$ from the screen.

5. Connect a PC computer to each test unit that will serve to run the test program. NOTE: On each trial, the test program determines the test regimen of the subjects (e.g., property of the display, stimulus duration, etc.) and associates the behavioral measures (e.g., response time, accuracy) to the monkey identified by the RFID technology.

6. Connect a food dispenser to the PC computer via a parallel port, as described in ${ }^{1}$. This dispenser delivers grains of dry wheat inside the ALDM monkey area.

7. Inject a RFID microchip in the forearm of each individual of the group as described in ${ }^{1}$. NOTE: This procedure should be performed according to the veterinarian practices.

A

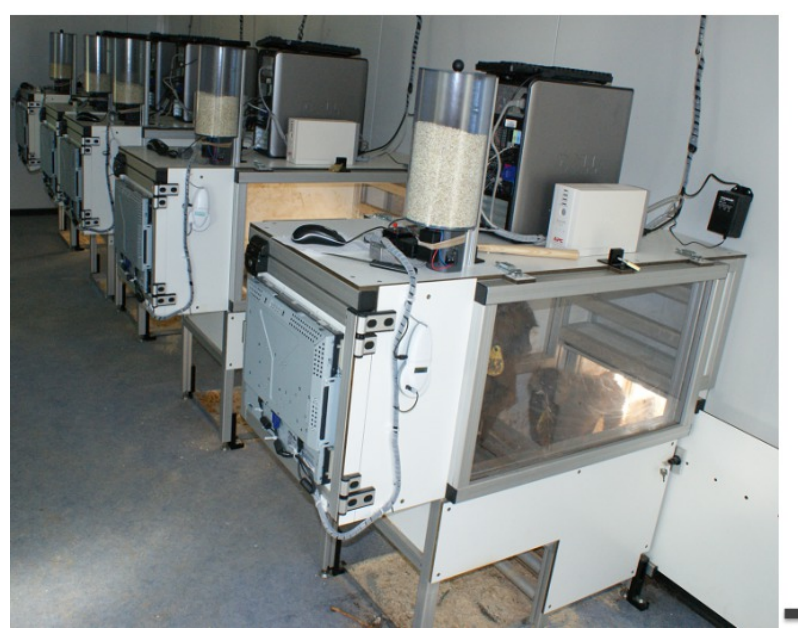

B

A

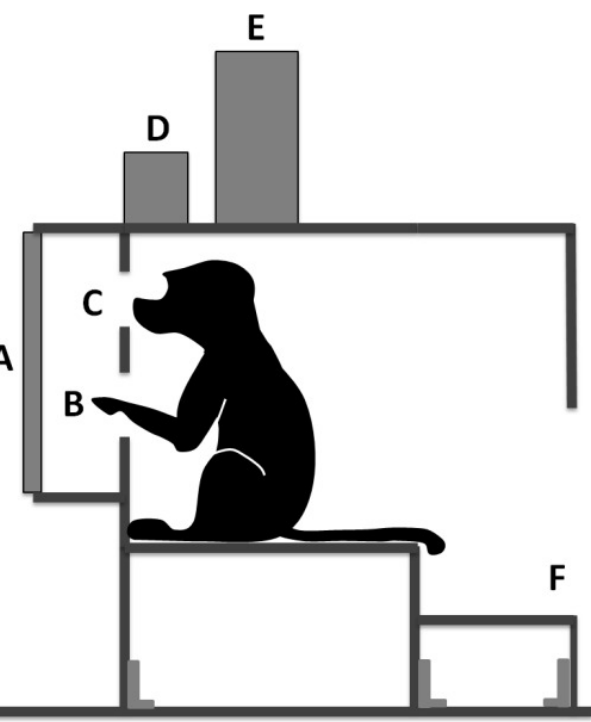

Figure 1: Picture (A) and schematic view (B) of an ALDM unit. Each test unit has five main component elements. (A) touchscreen; (B) handport; (C) viewport; (D) food dispenser delivering food items inside the cage after a correct response; (E) computer; (F) door providing access from the enclosure. Please click here to view a larger version of this figure.

\section{ALDM Network Set-up}

1. Install 10 ALDM units inside two $8 \times 4 \mathrm{~m}^{2}$ trailers, with 5 ALDM units in each trailer as described in ${ }^{1}$. Connect each ALDM test unit to a unique controlling server accessible via an IP address (see Figure 2). Ensure that the ALDM unit can both download and upload information from/to the server.

2. Write a computer testing program that downloads text files from the server once a monkey is identified by an ALDM test unit as per ${ }^{1}$. In the text file, provide indications on the current experimental status (number of trials already performed in the training or testing phase) of the monkey using the unit. Write the testing program such that it can use that information for presenting the training or test trial predefined by the experimental procedure.

3. Insert the test program inline script such that it uploads and downloads data from the server. Ensure that the ALDM unit synchronizes the ALDM clock twice a day. 
NOTE: With this procedure, the 10 ALDM units can provide indications on which monkey uses which unit, and when, and therefore provide information on the social dynamics in the use of the test systems.

4. Write the testing program such that before each trial, the test program uploads information from the server on the current experimental status of the monkey. Once the trial is complete, the ALDM unit will update the subject's text file on the server

NOTE: Centralization of the subject's related parameters on the controlling server guarantees that the participants will be tested in the predefined test order, independently of ALDM test units it decides to use.

5. Store the following data as a text file after each trial: Date, Day time (HH:MM:SS format), Name of the current test program, Monkey name, Number assigned to the ALDM test unit (e.g., 1), Test level (e.g., training), Score $(1=$ correct behavioral response; $2=$ incorrect response), Response time with msec precision.

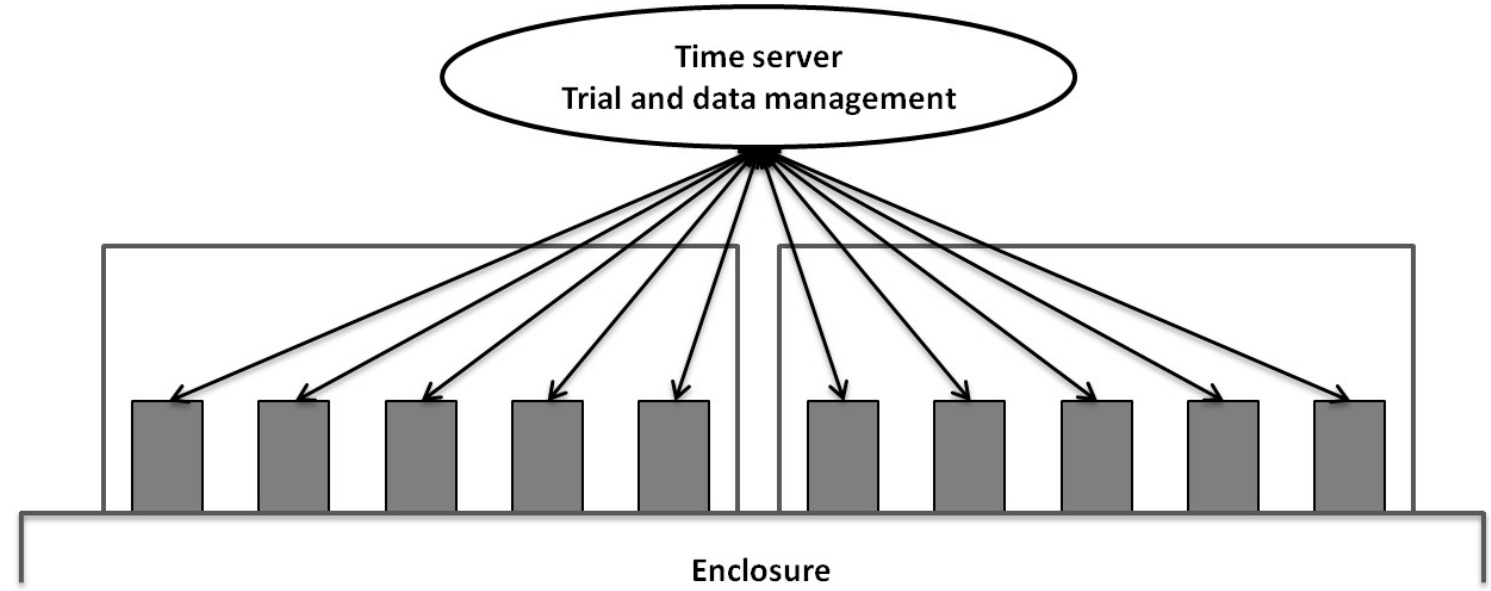

Figure 2: Schematic representation of the ALDM network. Each ALDM unit receives and provides information from/to a unique controlling server. Please click here to view a larger version of this figure.

\section{Illustration of the Use of the ALDM Network for the Study of Social Cognition}

NOTE: The ALDM protocol has the unique advantage of having monkeys that can interact freely with computers, while being maintained in their social group. Below are presented 3 protocols illustrating the use of a network of ALDM test units to collect unprecedented information on baboons' (Papio papio) social cognition.

1. Computerized ALDM testing combining behavioral (ethological) observations:

NOTE: The main goal of this research is to assess the effects of the valence (positive vs. negative) of affective states on the animals' cognitive performance during computerized ALDM testing. The procedure is outlined in Figure $\mathbf{3}$ and full details on this research were published in Marzouki et al. ${ }^{10}$

1. Install a visual search task consisting of the selection of a target visual stimulus among several distractors on each computer.

2. During eight days, observe and record manually which behavior each individual exhibits in the enclosure, and when, with a timer synchronized to the controlling server.

3. Group the behaviors exhibited in the enclosure in three clusters corresponding to the emotionally positive social events (e.g., grooming), the emotionally negative social events (e.g., threat), and the emotionally nonsocial (neutral) events (e.g., object manipulation).

4. Analyze the response times of the visual search task obtained within a 3 min time window following a behavioral observation in the enclosure. Synchronize the behavioral observations and computer data and analyze (see Figure 3).

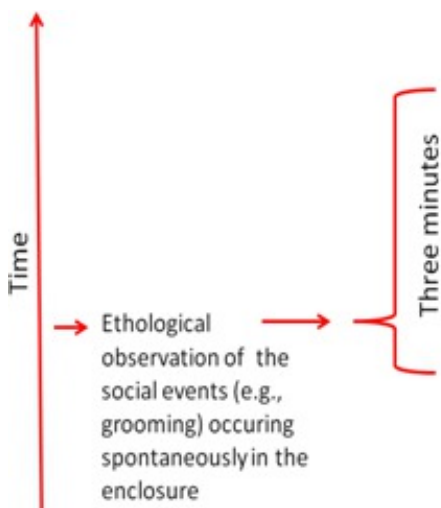

$$
\begin{aligned}
& \text { Recording of the } \\
& \text { performance (score } \\
& \text { and response times) } \\
& \text { during the three } \\
& \text { minutes following a } \\
& \text { recorded social event }
\end{aligned}
$$

Figure 3: Schematic illustration of Section 3.1. The spontaneous behaviors expressed in the enclosure are recorded in parallel with the cognitive performance on the ALDM tests.

2. Assessment of social influences during ALDM testing: 
NOTE: This protocol is aimed at studying audience effects on cognitive performance recorded during computerized ALDM testing. Full details on this research were published in Huguet et al. ${ }^{11}$

1. On each ALDM test unit, install a task in which a circle is followed by a response on the right side of the screen or on the left side when a parallelogram is presented.

2. Allow the monkey to use the 10 ALDM test systems as they want. Several monkeys can therefore use adjacent test systems. Record which individual uses which system.

NOTE: The baboons can see their conspecifics (when present) using the other test setups of the trailer, thanks to the transparent side walls of each unit.

3. Make sure that the test program records the performance of each individual, as well as the social context of the trial (i.e., if the other ALDM test units are simultaneously used, and by whom).

4. Analyze variations in response time depending on the social context.

3. Cultural evolution assessed via computerized transfer of information from individuals to individuals:

NOTE: This protocol was developed to study the evolution of information transmitted through a chain of participants. The procedure is outlined in Figure 4 and full details on this research were published in Claidière et al. ${ }^{12}$

1. Train the participants to solve a simple memory task in which they first see four randomly chosen red squares in a four by four grid of otherwise white ones. After $400 \mathrm{msec}$ change all the red squares to white, and ask the participant to touch the squares that were previously red.

2. When the training is completed, use the behavioral response of one participant (the squares touched) to produce the stimuli for the next individual in the chain (see Figure 4). Except for the first individual in the chain for which the grids are randomly generated by the computer.

3. Analyze the success at the task with the number of transmission events

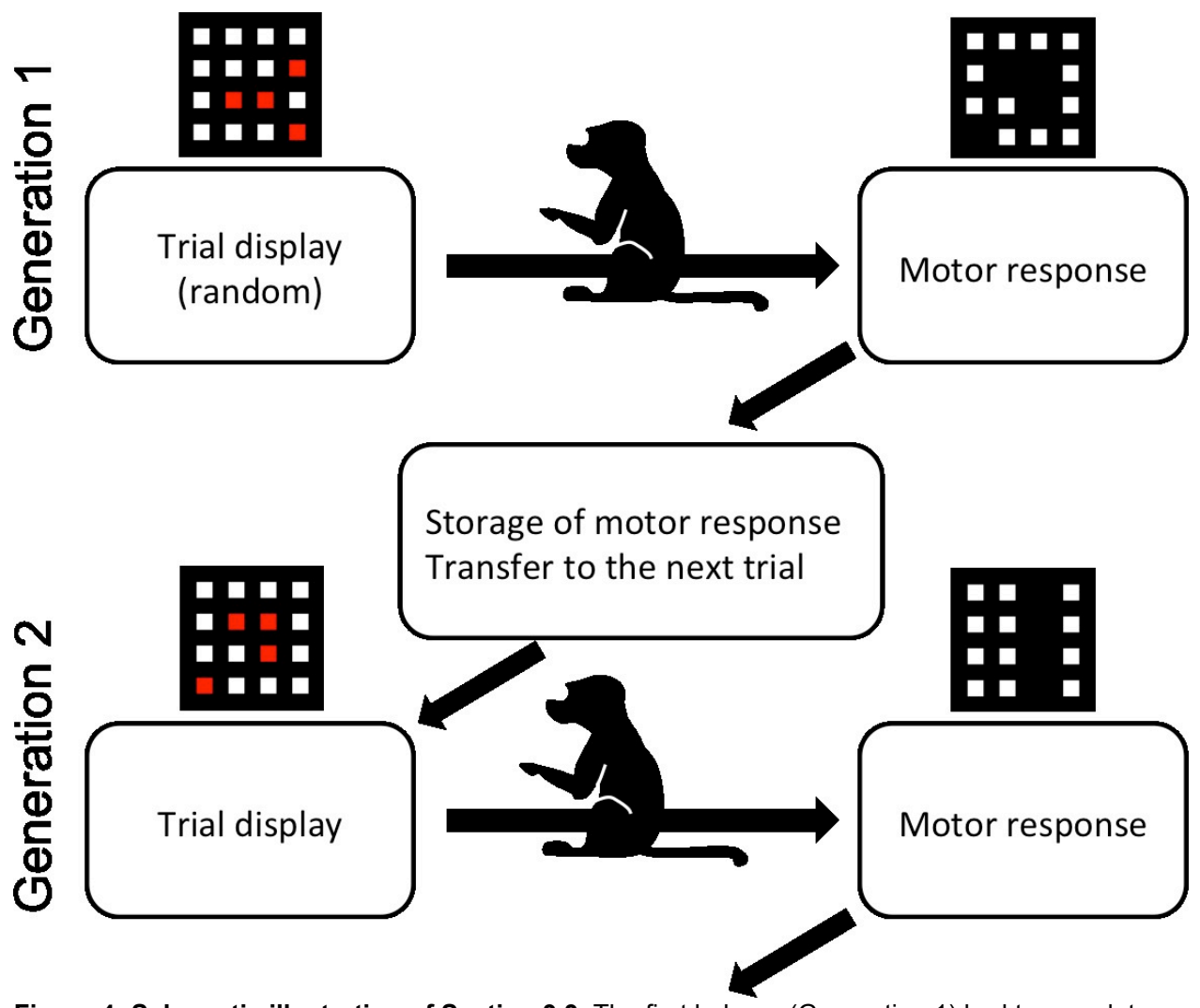

Figure 4: Schematic illustration of Section 3.3. The first baboon (Generation 1) had to complete a set of 50 trials generated by the computer. The motor responses of this baboon were then used to create the stimuli for the second baboon (Generation 2) in the transmission chain. This procedure was repeated until the last baboon in the chain was tested. Please click here to view a larger version of this figure.

\section{Representative Results}

As shown in Figure 5, the response times following negatively valenced behaviors, such as threats, were significantly slower than those following neutral (e.g., object manipulation), and positively valenced social behaviors, such as grooming. This study demonstrates that social moods affect the performance during cognitive testing. Interested readers will find more detailed information about this study in Marzouki et al. ${ }^{10}$ In Figure 6, compared to isolation, the presence of others reduced response times (the response time decreases with the number of individuals present in the trailer), indicating a social facilitation effect of well-learned responses. More detailed about this research can be found in Huguet et al. ${ }^{11}$ Figure 7 indicates an increase in performance during transmission trials (blue squares) but not in a control (random) condition (yellow circles). More detailed information on this research can be found in Claidière et al. ${ }^{12}$ 
590

585

580

575

570

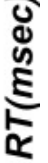

565

560

555

550

\section{Positive}

Negative

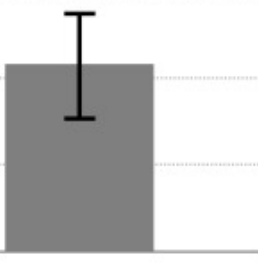

Neutral

Figure 5: Illustrative results from Section 3.1. Mean response times (msec) in the visual search task immediately following social positive, negative or neutral events. Error bars represent the standard deviation (SD).

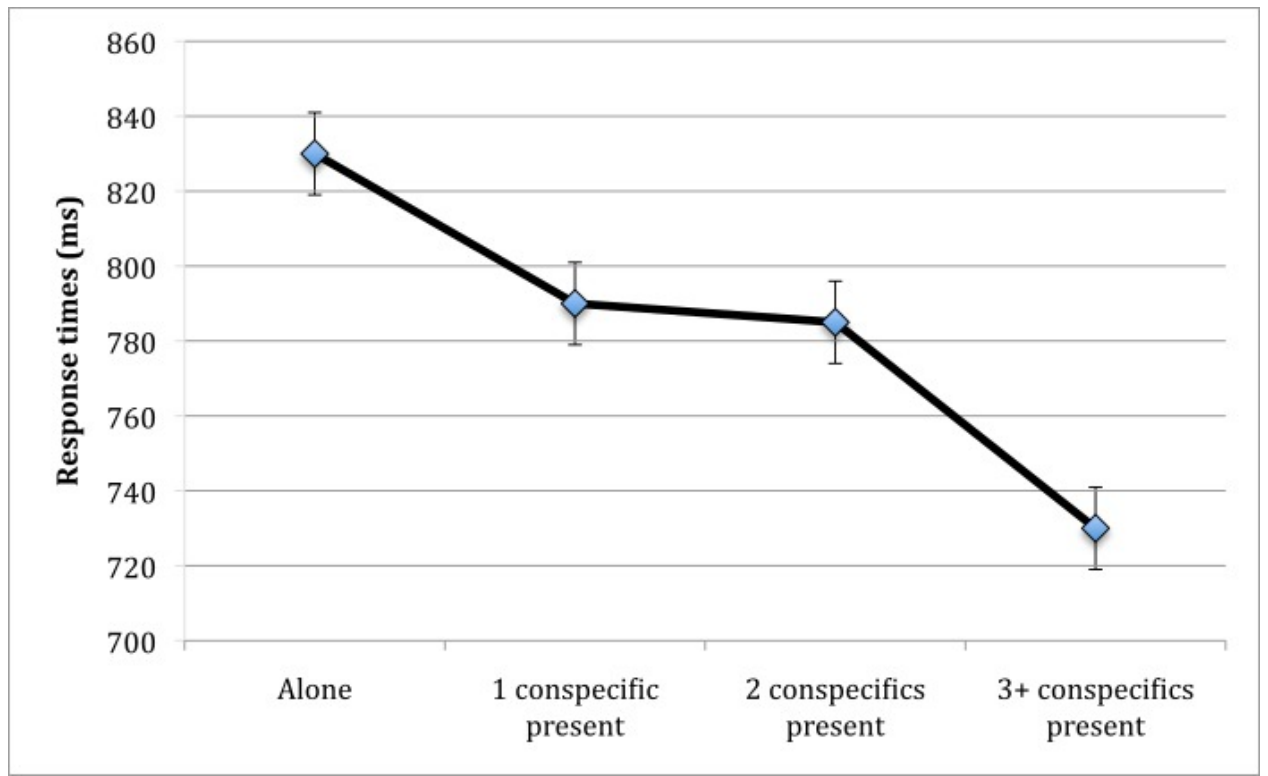

Figure 6: Illustrative results from Section 3.2. Social presence effect on the speed of well-learned responses. This figure shows the mean response times (msec) depending on the number of conspecifics present during each trial in the adjacent ALDM test units. Error bars represent the standard error (SE). 


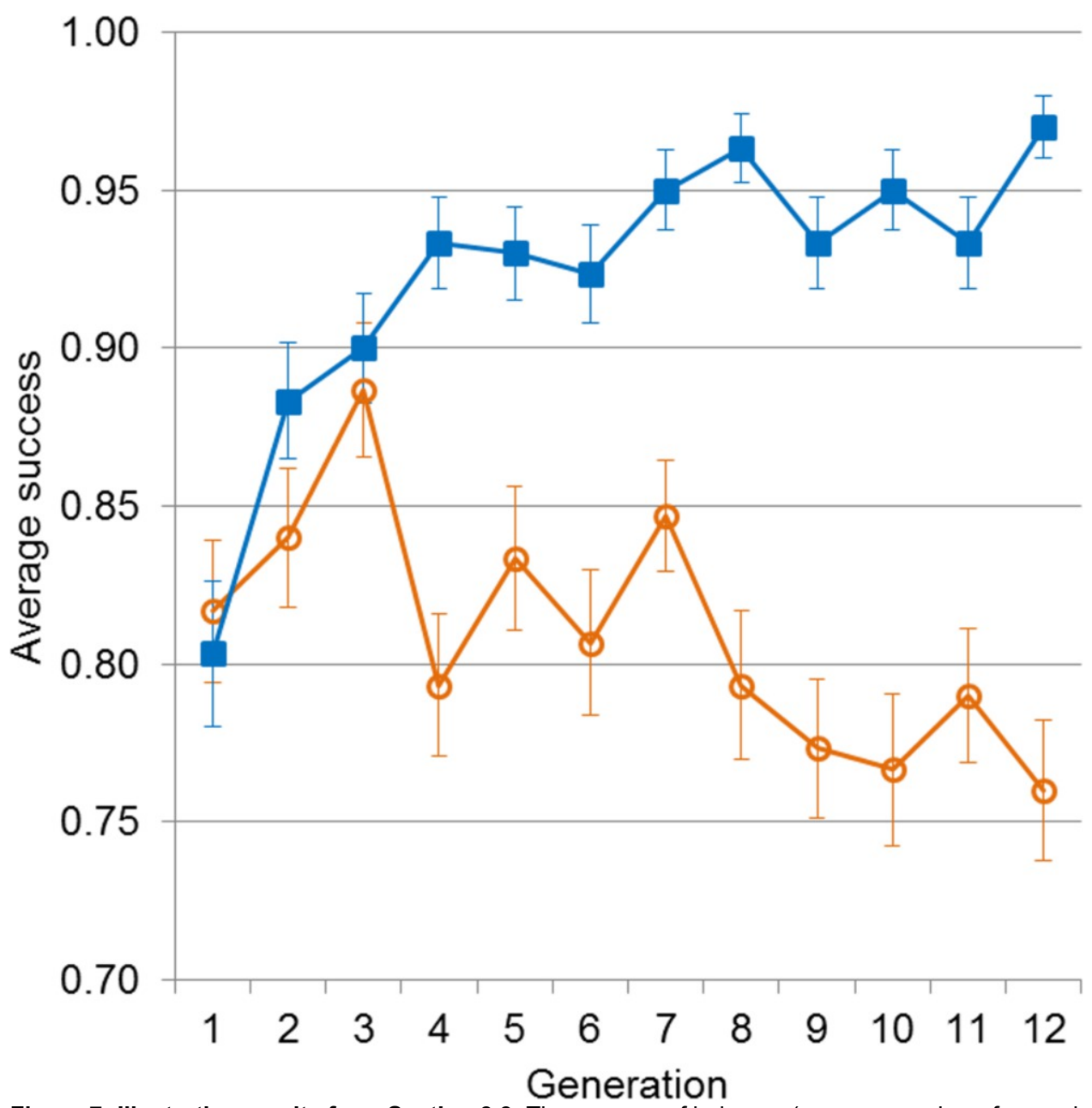

Figure 7: Illustrative results from Section 3.3. The success of baboons (average number of rewards for 50 trials) increased with the number of transmission events (generation) during transmission trials (blue squares) but not during random control trials (yellow circles). Error bars represent the SE.

\section{Discussion}

Primatologists have always expressed a strong interest in social and non-social aspects of cognition. However the integration of these two levels of understanding has been difficult. Social testing is sometimes conducted in laboratories, but on a very restricted number of subjects ${ }^{13}$ A network of ALDM units offers 4 main advantages in that respect: (1) ALDM testing uses highly sophisticated (computerized) protocols and millisecond precision measurements of the cognitive processes; (2) ALDM testing is achieved within the natural social context of the studied animals; (3) ALDM testing can be proposed to large groups, as done in our studies; (4) ALDM testing takes advantage of the spontaneous occurrence of social interactions and do not constrain the individuals to particular social situations.

ALDM network therefore opens avenues for integrative and multidimensional analyses of cognition combining social and non-social factors.

This paper illustrates this integrative approach using three protocols but many other possibilities exist beside these key illustrations. For instance, preliminary (unpublished) evidence collected in our laboratory suggests that the monkeys using adjacent test units also have the strongest affiliative bonds within their social group. If this effect is confirmed, then the automaticity of ALDM testing will allow the collection of large amount of information on social dynamics that can facilitate longitudinal sociometric studies (e.g., based on measures of social distance) in non-human primates.

The limitations of ALDM testing remain unknown at this stage. For instance, it is still unknown to what extent the activity level of the subjects can be influenced by the social structure (e.g., sex ratio) of the group. Nevertheless, ALDM testing seems a highly promising tool to bridge the apparent gap between studies on individual cognition and those more focused on social cognition. Its use offers the opportunity to identify the social factors that may influence individual cognition, and to more precisely measure their effects. Furthermore, our previous research has shown that ALDM testing also promotes animal welfare: They are forms of behavioral enrichments that reduce stress levels (inferred from cortisol level and the number of abnormal stereotypies) $)^{3}$. Accordingly, ALDM testing seems highly beneficial for both scientists and animals, and should be more extensively used in the future in laboratories and zoos. 


\section{Disclosures}

The authors declare that no competing interests exist.

\section{Acknowledgements}

This research was supported by the "Federation de Recherche 3C", Aix-Marseille University and the EU Euprim-Net-II project (\#RII3-026155). This work was supported by a public grant overseen by the French National Research Agency (ANR) as part of the programs LICORNES (ANR-12-CULT-0002) and ASCE (ANR-13-PDOC-0004). The staff of the Rousset-sur-Arc CNRS primate station is acknowledged for technical support.

\section{References}

1. Fagot, J., \& Paleressompoulle D. Automatic testing of cognitive performance in baboons maintained in social groups. Behav Res Methods. 41 (2), 396-404, doi: 10.3758/BRM.41.2.396 (2009).

2. Fagot, J., \& Bonté, E. Automated testing of cognitive performance in monkeys: Use of a battery of computerized test systems by a troop of semi-free ranging baboons. Behav Res Methods. 42 (2), 507-516, doi: 10.3758/BRM.42.2.507 (2010).

3. Fagot, J., Gullstrand, J., Kemp, C., Defilles, C., \& Mekaouche, M. Effects of freely accessible computerized test systems on the spontaneous behaviors and stress level of Guinea baboons (Papio papio). Am J Primatol. 76 (1), 56-64, doi: 10.1002/ajp.22193 (2014).

4. Dréa, C. M. Studying primate learning in group contexts: Tests of social foraging, response to novelty, and cooperative problem solving. Methods. 38 (3), 162-177, doi:10.1016/j.ymeth.2005.12.001 (2006).

5. Grainger, J., Dufau, S, Montant, M, Ziegler, J.C \& Fagot, J. Orthographic processing in baboons. Science. 336 (6078), 245-248, doi: 10.1126/ science.1218152 (2012).

6. Fagot, J., \& Thompson, R.K.R. Generalized Relational Matching by Guinea Baboons (Papio papio) in two by two-item analogy problems. Psychol Sci. 22 (10), 1304-1309, doi: 10.1177/0956797611422916 (2011).

7. Whitehouse, J., Micheletta, J., Powell, L.E., Bordier, C., Waller, B.M. The Impact of Cognitive Testing on the Welfare of Group Housed Primates. PLOS ONE. 8 (11), e78308. doi: 10.1371/journal.pone.0078308 (2013).

8. Gazes, P.R, Brown, E.K., Basile, BM., Hampton, R.R. Automated cognitive testing of monkeys in social groups yields results comparable to individual laboratory-based testing. Anim Cogn. 16 (3), 445-458 ,doi:10.1007/s10071-012-0585-8 (2012).

9. Huber, L.Heise, N, Zenan, C., \& Palmers, C. The ALDB box: Automatic testing of cognitive performance in groups of aviary-housed pigeons. Behav Res Methods. Apr 16. [Epub ahead of print], doi: 10.3758/s13428-014-0462-2 (2014).

10. Marzouki, YB., Gullstrrand, J., Goujon, A. \& Fagot, J. Baboons' response speed is biased by their moods. PLoS One. 9 (7), e102562, doi: 10.1371/journal.pone.0102562, (2014).

11. Huguet, P., Barbet, I., Belletier, C., Monteil, J.M., \& Fagot, J. Cognitive control under social influence in baboons. Journal of Experimental Psychology : General. (2014).

12. Claidière, N., Smith, K., Kirby, S., \& Fagot, J. Cultural evolution of systematically structured behaviour in a non-human primate. Proc R Soc. Lond. B. 281 (1797), doi: 10.1098/rspb.2014.1541 (2014).

13. Subiaul, F., Cantlon, J.F., Holloway, R.L., Terrace, H.S. Cognitive imitation in Rhesus macaques. Science. 305 (5682), 407-410, doi: 10.1126/ science.1099136 (2004). 